\title{
Unveiling the dynamics of optical frequency combs from phase-amplitude correlations
}

\author{
M. Ansquer, ${ }^{1}$ V. Thiel, ${ }^{2}$ S. De $\odot,{ }^{3}$ B. Argence, ${ }^{1}$ G. Gredat $\odot,{ }^{4}$ F. Bretenaker $\odot,{ }^{4}$ and N. Treps ${ }^{1, "}$ \\ ${ }^{1}$ Laboratoire Kastler Brossel, Sorbonne Université, ENS-Université PSL, CNRS, Collège de France, 4 Place Jussieu, 75252 Paris, France \\ ${ }^{2}$ Department of Physics and Oregon Center for Optical, Molecular, and Quantum Science, University of Oregon, Eugene, Oregon 97403, USA \\ ${ }^{3}$ Integrated Quantum Optics Group, Applied Physics, 100 Warburger Straße, Paderborn University, Paderborn 33098, Germany \\ ${ }^{4}$ Université Paris-Saclay, CNRS, ENS Paris-Saclay, CentraleSupélec, LuMIn, 91190 Gif-sur-Yvette, France
}

(Received 1 March 2021; accepted 6 July 2021; published 26 July 2021)

\begin{abstract}
The noise dynamics of an optical frequency comb based on a mode-locked Ti-sapphire laser is analyzed in terms of noise modes. A spectrally resolved multipixel homodyne detection enables the simultaneous measurement of the amplitude and phase noises of several optical frequency channels, from which the covariance matrices of the amplitude and phase quadratures of the laser field are calculated. The decomposition of these matrices into the four most significant time/frequency modes of the field enables the tracking of the origin of the noises and the correlations between the noise modes. In particular, the correlations between the amplitude and phase noises are measured. These measurements are well reproduced by a model taking into account the correlations between the carrier envelope offset phase noise and the amplitude noise induced by the group velocity dispersion of the laser cavity.
\end{abstract}

DOI: 10.1103/PhysRevResearch.3.033092

\section{INTRODUCTION}

Mode-locked femtosecond lasers, or optical frequency combs (OFCs), have become a ubiquitous tool in metrology for the past 20 years. They were originally used to transfer the excellent spectral properties of the optical atomic clocks to the microwave frequency domain $[1,2]$. They have now found applications in numerous fields, such as tests of fundamental physics [3-6], atomic and molecular spectroscopy [7,8], time or frequency transfer [9-11], ranging measurements [12,13], and astrophysics [14]. Many of those applications rely on the stability of the OFC. Furthermore, understanding the dynamics of OFCs, i.e., the noises affecting them, is critical to develop ultrastable sources so they do not limit the precision of the measurement.

For a single frequency laser, the dynamics is described in terms of amplitude noise (i.e., variation in the photon number) and phase noise (i.e., variation in frequency). However, for an OFC, composed of roughly $10^{5}$ spectral lines, amplitude and phase noises affect each of them individually. Thus, such a complete characterization is almost impossible due to the gigantic number of degrees of freedom. Yet, it has been theorized that the main dynamics of an OFC can be reduced to four distinct parameters: the pulse energy, the carrier envelope offset (CEO), the repetition rate, and the central wavelength $[15,16]$, indicating the presence of correlations

\footnotetext{
*nicolas.treps@1kb.upmc.fr

Published by the American Physical Society under the terms of the Creative Commons Attribution 4.0 International license. Further distribution of this work must maintain attribution to the author(s) and the published article's title, journal citation, and DOI.
}

between the different spectral lines. Each parameter is associated to a specific time/frequency mode of the electric field. Thus, investigating those spectral correlations can give access to information on the laser dynamics. Spectral correlations have started to draw attention for various applications such as to track the Raman response of materials [17], for example, or as in Ref. [18]. In that study, Brajato et al. used those correlations to measure amplitude and phase noise correlation matrices with Bayesian filtering for a frequency comb.

On the other hand, the dynamics of OFCs has been widely studied by measuring the noise on each parameter separately. A complete description of the noise in passively mode-locked lasers was developed by Haus and Mecozzi [16] and later generalized by Paschotta et al. [19-21]. A thorough understanding of the dynamics of mode-locked fiber lasers is given in Refs. [22,23], both theoretically and experimentally. The intensity-related dynamics of OFCs has drawn a lot of attention. Changes in the lasers parameters under pump laser modulation is studied in Refs. [24-26]. Those studies indicate that the main source of noise is the intensity fluctuations of the pump laser. They are responsible for several features such as frequency pulling, timing jitter, and phase noise.

In previous studies, different setups were used to access the noise of the different laser parameters. Characterizing the intensity noise is the easiest task, as a single photodiode is sufficient. The CEO noise is usually characterized by beating the comb spectrum with its frequency-doubled counterpart. The spectrum must be expanded beforehand to cover an octave [27]. The timing jitter can be measured by various techniques such as heterodyne beat with an ultrastable laser [28] or by self-heterodyne with a fiber delay line interferometer [29,30]. Furthermore, most of the studies concentrate on the lower part of the noise spectrum, from the $\mathrm{Hz}$ to tenth of $\mathrm{kHz}$. In this frequency range, technical noises are dominant. 
In this paper, building on the experimental scheme introduced in Ref. [31] based on the investigation of spectral correlations, we present an experiment to measure with a single setup the four noise parameters at the quantum limit, enabling the full characterization of the laser dynamics. To do so, a spectrally resolved multipixel homodyne detection is used to measure the covariance matrices of the amplitude and phase quadratures of the field. The fluctuations of the laser parameters are recovered by extraction of the noise in the corresponding time/frequency mode from those covariance matrices. We focus our analysis on the frequency range of the noise spectrum from $200 \mathrm{kHz}$ to $4 \mathrm{MHz}$. This range has drawn less attention in the literature. As a matter of fact, most of the investigations of frequency comb dynamics are for metrological applications. Those studies focus on the lower frequency range (a few to a hundred hertz), corresponding to the long-term stability of the experiment, where the technical noises dominate. However, at higher frequencies, the laser is expected to reach the quantum limit and can thus be used for precise measurements. In this paper, the laser is found to be shot noise limited around $3 \mathrm{MHz}$. In addition, the simultaneous measurement of the amplitude and phase quadratures gives access to correlations between amplitude and phase noises. The resulting amplitude-phase correlation matrices can be studied through singular value decomposition (SVD). Thus, we confirm that those correlations are a signature of the intensity-related dynamics of the laser. We analyze this dynamics thanks to a simple model explaining the intensity dependence of the CEO frequency. By comparing the measured CEO frequency noise and the model, we show that in our laser, the CEO intensity dynamics is induced by the spectrum center frequency fluctuations via the group velocity dispersion (GVD) of the laser cavity.

\section{MODAL DESCRIPTION OF THE NOISE}

\section{A. The perturbed pulse}

Our approach consists of investigating the noise of a single pulse from an OFC whose complex electric field is written as

$$
E(t)=\mathcal{E}_{0} a(t) e^{-i \omega_{c} t},
$$

where $\mathcal{E}_{0}$ is the single photon field amplitude [32], $\omega_{c}$ is the carrier frequency, and $a(t)$ the slowly varying Gaussian envelope of the pulse. This analysis can then easily be extended to the train of pulses emitted by an OFC according to

$$
E_{\mathrm{comb}}(t)=\sum_{n} E\left(t-n \tau_{r}\right) e^{-\mathrm{in} \Delta \phi_{\mathrm{CEO}}},
$$

where $\tau_{r}$ is the repetition rate of the laser and $\Delta \phi_{\text {CEO }}$ is the CEO phase.

The laser pulses undergo intensity and phase noise due to various sources such as pump laser intensity fluctuations, vibrations, or temperature fluctuations. In most cases, the resulting dynamics of the laser can be reduced to four parameters, $\vec{p}=\left(\delta \epsilon, \delta \omega_{c}, \delta \tau_{\mathrm{CEO}}, \delta \tau_{r}\right)$ where $\delta \epsilon$ stands for amplitude fluctuations, $\delta \omega_{c}$ for carrier frequency fluctuations, $\delta \phi_{\mathrm{CEO}}=\omega_{c} \delta \tau_{\mathrm{CEO}}$ for CEO phase fluctuations, and $\delta \tau_{r}$ of the timing jitter. Note that $\delta \phi_{\mathrm{CEO}}$ represents the fluctuations of the CEO phase and should formally be noted $\delta \Delta \phi_{\text {CEO }}$. This notation being too heavy we keep $\delta \phi_{\mathrm{CEO}}$ to represent the CEO fluctuations.

Thus, the starting point of our modal description of the dynamics is to study a single pulse Eq. (1) undergoing a perturbation of those four parameters [33]. The perturbed pulse is therefore written

$$
E(t, \vec{p})=\mathcal{E}_{0}(1+\delta \epsilon) a\left(t-\delta \tau_{r}\right) e^{-i\left(\omega_{c}-\delta \omega_{c}\right)\left(t-\delta \tau_{\mathrm{CEO}}\right)} .
$$

The same expression can be obtained in the spectral domain by taking the Fourier transform of the previous expression,

$$
E(\Omega, \vec{p})=\mathcal{E}_{0}(1+\delta \epsilon) a\left(\Omega-\delta \omega_{c}\right) e^{i\left(\omega_{c} \delta \tau_{\mathrm{CEO}}+\Omega \delta \tau_{r}\right)},
$$

where $\Omega=\omega-\omega_{c}$. As those fluctuations are small, the above expression can be expanded at first order to obtain the fluctuating electric field,

$$
\begin{aligned}
\delta E(\Omega)= & E(\Omega, \vec{p})-E(\Omega) \\
\simeq & \mathcal{E}_{0}\left[\delta \epsilon a(\Omega)-\delta \omega_{c} \frac{\partial a(\Omega)}{\partial \Omega}\right. \\
& \left.+i \omega_{c} \delta \tau_{\mathrm{CEO}} a(\Omega)+i \Omega \delta \tau_{r} a(\Omega)\right] .
\end{aligned}
$$

Note that the notation $\delta$ as in $\delta E(\Omega)$ implies the dependence in $\vec{p}$, i.e., $\delta E(\Omega)=\delta E(\Omega, \vec{p})$. In the following, we write $a(\Omega)=\alpha(\Omega) e^{i \phi(\Omega)}=\alpha_{0} u(\Omega) e^{i \phi(\Omega)} . \alpha_{0}$ is the field amplitude so $\alpha_{0}=\sqrt{N_{0}}$ with $N_{0}$ the mean photon number, $u(\Omega)$ is the mean-field mode normalized as $\int d \Omega|u(\Omega)|^{2}=1$ and $\phi(\Omega)$ the spectral phase. For simplicity, we choose it to be constant over $\Omega$ and equal to zero, i.e., $e^{i \phi(\Omega)}=1$. Without this simplification, the definition of the quadratures, introduced in the next section, as the real and imaginary parts of the field proportional to the amplitude and the phase, would be less straightforward.

\section{B. Field quadrature fluctuations}

The field fluctuations can also be written in terms of quadratures of the electric field $x(\Omega)$ and $p(\Omega)$, respectively, proportional to the real and imaginary parts of the field defined by

$$
E(\Omega)=\frac{\mathcal{E}_{0}}{2}[x(\Omega)+i p(\Omega)] .
$$

Thus, we have

$$
2 \delta E(\Omega)=\mathcal{E}_{0}[\delta x(\Omega)+i \delta p(\Omega)],
$$

with $\delta x(\Omega)=2 \delta \alpha(\Omega)$, proportional to the amplitude fluctuations, and $\delta p(\Omega)=2 \alpha(\Omega) \delta \phi(\Omega)$, proportional to the phase fluctuations. By identification with Eq. (5), we find

$$
\begin{gathered}
\delta x(\Omega)=2 \alpha_{0}\left[\delta \epsilon u(\Omega)-\delta \omega_{c} \frac{\partial u(\Omega)}{\partial \Omega}\right], \\
\delta p(\Omega)=2 \alpha_{0}\left[\omega_{c} \delta \tau_{\mathrm{CEO}} u(\Omega)+\Omega \delta \tau_{r} u(\Omega)\right] .
\end{gathered}
$$

In the expressions above, each of the four parameters is associated to a particular spectral mode related to the mean-field one. Let us consider a Gaussian mean-field spectral mode $u(\Omega)=\frac{1}{\sqrt[4]{2 \pi \Delta \omega^{2}}} \exp \left(-\frac{\Omega^{2}}{4 \Delta \omega^{2}}\right)$, where $\Delta \omega$ is the spectral width 


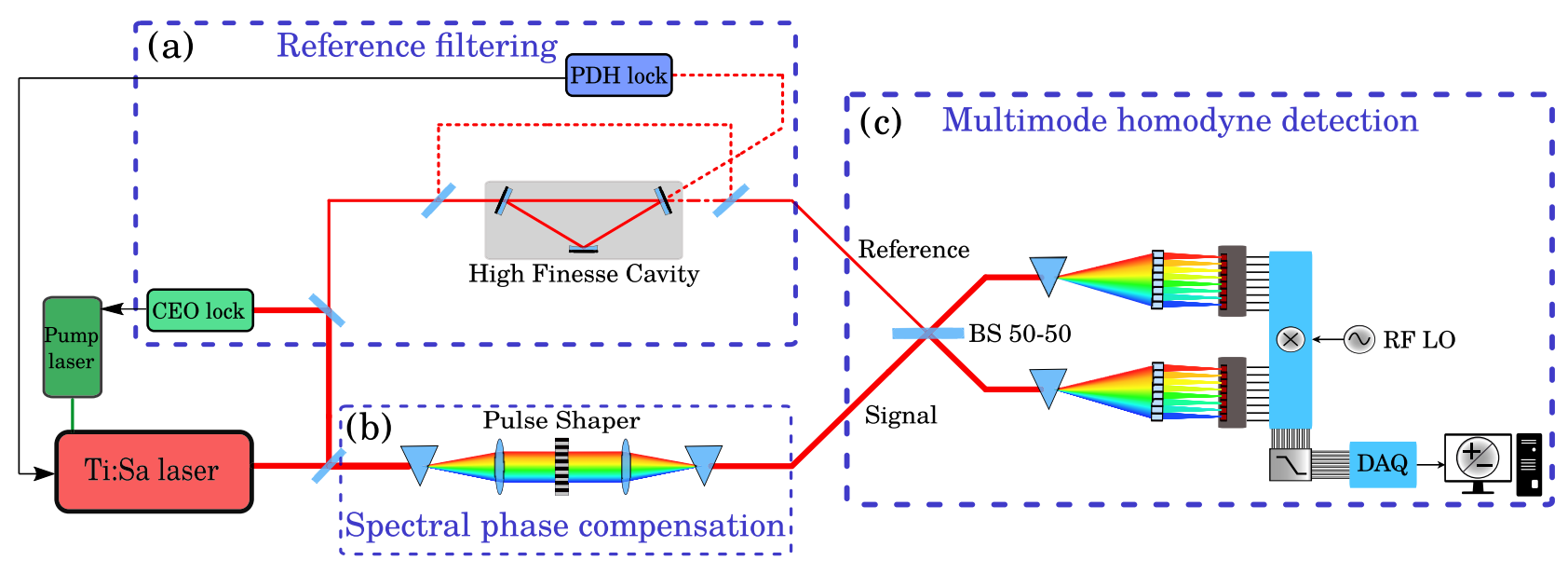

FIG. 1. Experimental setup: The experiment is composed of three parts. (a) Reference filtering, to generate the reference beam via a high finesse cavity on which the repetition rate of the laser is locked. The CEO is also locked thanks to an $f$ - $f f$ interferometer to ensure maximum transmission through the cavity. The laser is free running above the locking bandwidth of a few kHz. (b) Spectral phase compensation with a pulse shaper to ensure a flat phase for all spectral bands. That way, the homodyne is locked on the phase quadrature for all the spectral bands. (c) Multipixel homodyne detection to measure the amplitude and phase noise in eight different spectral bands. A demodulation stage is used to acquire noise at a given offset frequency selected by the radio-frequency local oscillator (RF LO) whose frequency can be swept. All data are acquired by an acquisition card (DAQ) and postprocessed on a computer to recover the covariance matrices.

of the field given by $\Delta \omega^{2}=\int d \Omega^{2}|u(\Omega)|^{2}$. The quadratures Eqs. (8) and (9) can finally be written as

$$
\delta x(\Omega)=2 \alpha_{0}\left[\delta \epsilon u_{\mathrm{amp}}(\Omega)-\frac{\delta \omega_{c}}{2 \Delta \omega} u_{\mathrm{cent}-\mathrm{freq}}(\Omega)\right],
$$

$$
\delta p(\Omega)=2 \alpha_{0}\left[\omega_{c} \delta \tau_{\mathrm{CEO}} u_{\mathrm{CEO}}(\Omega)+\Delta \omega \delta \tau_{r} u_{\text {rep-rate }}(\Omega)\right],
$$

with $\quad u_{\text {amp }}(\Omega) \equiv u_{\mathrm{CEO}}(\Omega)=u(\Omega), \quad u_{\text {cent }- \text { freq }}(\Omega)=$ $-2 \Delta \omega \frac{\partial u(\Omega)}{\partial \Omega}$, and $u_{\text {rep-rate }}(\Omega)=\frac{\Omega u(\Omega)}{\Delta \omega}$. Those normalized spectral modes are named detection modes.

In conclusion, the dynamics of the laser can be recovered by accessing spectral modes in the amplitude and phase quadratures of the electric field. As a consequence, a spectrally resolved detection of the quadratures is needed. This is provided by a multipixel homodyne detection described in the next section.

\section{MEASURING THE MULTIMODE FIELD}

The experiment is based on a spectrally resolved homodyne detection represented in Fig. 1(c). Note that the homodyne detection is not implemented in a standard way. As will be seen later, we actually measure the noise of the more intense of the two interfering beams while the weaker one serves as a reference. This will have consequences later on the sensitivity of the measurement. After the beam splitter (BS), the laser spectrum is split in eight spectral slices thanks to a grating and an array of microlenses. Two detectors, composed of eight photodiodes each, can then measure the amplitude and phase quadratures in each of those spectral bands. Consequently, the quadratures are measured in a pixelized basis with each pixel corresponding to a frequency band. We define the pixel basis $\left\{v_{i}(\Omega)\right\}, i \in\{1: 8\}$ with

$$
v_{i}(\Omega)= \begin{cases}u\left(\Omega_{m}\right) & \text { if } \Omega_{i} \leqslant \Omega<\Omega_{i+1} \\ 0 & \text { otherwise }\end{cases}
$$

with $\Omega_{m}=\left(\Omega_{i}+\Omega_{i+1}\right) / 2$.
The $x$ and $p$ quadratures of the field in this basis can be described by vectors $\vec{x}=\left(x_{1}, \ldots, x_{8}\right), \vec{p}=\left(p_{1}, \ldots, p_{8}\right)$ with

$$
\begin{aligned}
& x_{i}=\int v_{i}(\Omega) x(\Omega) d \Omega, \\
& p_{i}=\int v_{i}(\Omega) p(\Omega) d \Omega .
\end{aligned}
$$

This scheme allows us to measure the spectral covariance matrices in amplitude $\Gamma^{x}$ with $\Gamma_{i j}^{x}=\left\langle\delta x_{i} \delta x_{j}\right\rangle$ and in phase $\Gamma^{p}$ with $\Gamma_{i j}^{p}=\left\langle\delta p_{i} \delta p_{j}\right\rangle$ from which the dynamics of the laser can be extracted. The following section presents in detail the experimental setup used and represented in Fig. 1.

\section{A. Experimental setup}

The laser under study is a titanium-sapphire based femtosecond oscillator from Femtolasers company. It delivers 22 fs FWHM pulses with a repetition rate of $156 \mathrm{MHz}$, resulting in a $40 \mathrm{~nm}$ FWHM wide spectrum centered at $795 \mathrm{~nm}$. The average power is of the order of $1 \mathrm{~W}$. This laser is pumped by a $5 \mathrm{~W}$ Finesse Pure CEP pump laser at $532 \mathrm{~nm}$ from Laser Quantum. The beam under study is first split in two: an intense beam, the signal and a weak one, the reference. The reference is filtered by a high finesse cavity $(F \simeq 1200)$ to decorrelate the high frequency phase noises of the two beams. This cavity acts as a low pass filter for the phase fluctuations with a bandwidth of approximately $125 \mathrm{kHz}$. To ensure maximum transmission through the cavity, the CEO frequency and the repetition rate of the laser are locked. To do so, the CEO frequency is first measured thanks to an $f-2 f$ interferometer and mixed with a radio-frequency reference signal to obtain an error signal. This signal is then used to stabilize the CEO frequency by acting on the pump laser current via a commercial PI servo controller from New Focus with a bandwidth of $3 \mathrm{kHz}$. The repetition frequency is 
(a)

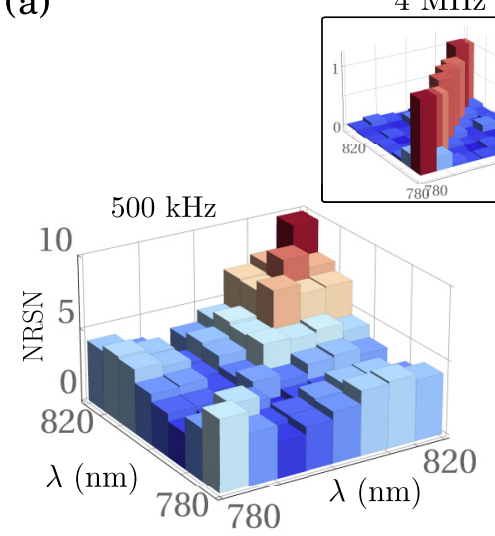

(b)

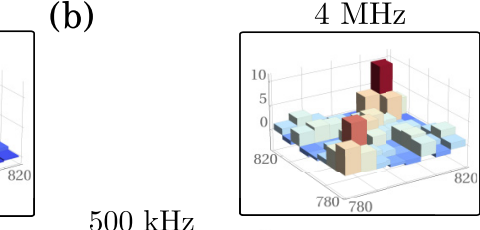

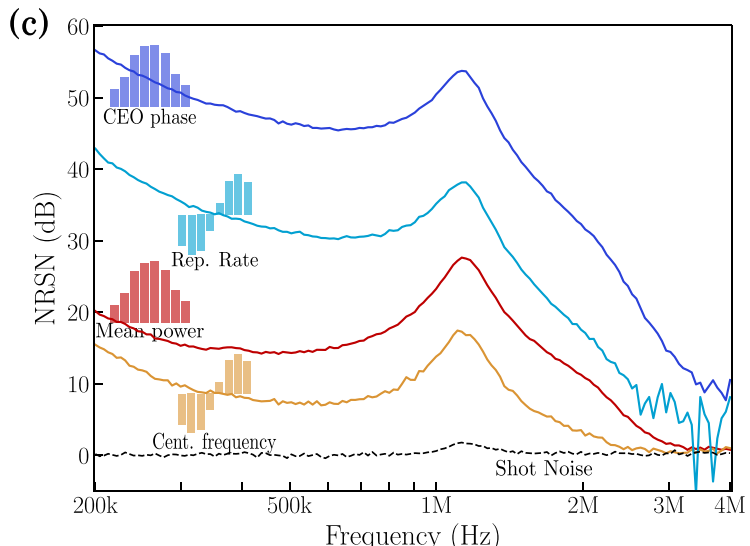

FIG. 2. Experimental covariance matrices. (a) Amplitude and (b) phase covariance matrices. The matrices are measured at $500 \mathrm{kHz}$. The noise is expressed as noise relative to shot noise (NRSN) on a linear scale. Correlations can be seen between the different spectral bands. In inset, matrices for noises at $4 \mathrm{MHz}$. At that frequency, the laser is only affected by the shot noise. (c) Noise on the physical parameters relative to the shot noise. Projection of the covariance matrices on the modes corresponding to the noise on the CEO, central, and repetition rate frequencies as well as the mean power. The noises are expressed in $\mathrm{dB}$ relative to the shot noise. The shot noise is measured by blocking the reference beam and taking the difference of the photocurrents.

stabilized by locking the laser cavity length on the high finesse cavity via a Pound-Drever-Hall (PDH) locking scheme. The PDH signal is derived from the reflected beam at the output of the cavity to avoid any modulation on the beam used for the detection. The error signal is fed to a mirror mounted on a piezoelectric actuator in the laser cavity via another PI servo controller with a bandwidth of $1 \mathrm{kHz}$. A detailed description of this locking scheme can be found in Ref. [31]. As both locks have a bandwidth of a few $\mathrm{kHz}$, the laser is thus free running above those frequencies. Spectral phase compensation using a pulse shaper is used to ensure a flat phase across the 40-nm spectrum of the signal beam at the detection. This compensation is needed to satisfy the assumption made in Sec. II A, namely, that $\phi(\Omega)$ is constant over $\Omega$. The reason for this is that if the phase were not flat, the nature of the measured quadratures would evolve throughout the optical spectrum. The phase compensation is performed by a spatial light modulator LCOS-SLM X10468 (liquid crystal on silicon) from Hamamatsu in a $4 f$ configuration [34]. The reference and signal beams are then recombined on a 50-50 BS. Finally, the spectrum is spatially spread and sent to two multipixel detectors thanks to arrays of microlenses. Each detector is composed of eight photodiodes. The detected signals are split into a low frequency component (dc) and a high frequency one (ac), with a cutoff frequency around $200 \mathrm{kHz}$. The dc part is used for alignment purpose and to phase lock the homodyne detection on the phase quadrature. The 16 ac signals are mixed with a reference signal delivered by a frequency generator whose frequency is swept, and low pass filtered with a cutoff frequency of $10 \mathrm{kHz}$. The resulting signals are then acquired by a data acquisition card NIPXIe 6361 from National Instrument with a sampling rate of $100 \mathrm{kSa} / \mathrm{s}$. The demodulation stage is used to measure noises at higher frequencies than the $1 \mathrm{MHz}$ bandwidth of the acquisition card but also to prevent the saturation of its dynamics. The laser being very noisy at low frequencies, acquiring all the noise spectrum in a single measurement would degrade the resolution of the spectrum at high frequencies where the noise is much lower.

This scheme allows us to measure the covariance matrices at a given offset frequency set by the frequency of the demodulating signal. The data are then processed by a computer. This processing and normalization procedure to retrieve the covariance matrices are detailed in the Appendices. Note that since the photocurrents of both detectors are measured, both the amplitude and the phase can be recovered simultaneously. This simultaneous measurement is a cornerstone of our work, as it allows the investigation of the correlations between amplitude and phase, one of the fundamental mechanisms that governs mode locking.

\section{B. Amplitude and phase covariance matrices}

An example of matrices acquired this way is shown in Fig. 2. Figures 2(a) and 2(b) reproduce the covariance matrices for the amplitude and phase fluctuations, respectively, for an offset frequency of $500 \mathrm{kHz}$. The inset displays the same matrices for an offset of $4 \mathrm{MHz}$. The matrices are expressed in units of shot noise (noise relative to shot noise, NRSN), which is the standard quantum limit in sensitivity for amplitude and phase noise. The shot noise is a white and uncorrelated noise corresponding to the level of noise associated to a coherent state. Therefore, the level of noise displayed by the covariance matrices must be understood as an excess of noise compared to a coherent state of the same mean power. The shot noise is measured experimentally by blocking the reference beam and measuring only the signal beam with the homodyne system. It is represented in Fig. 2(c) as a function of the frequency.

The amplitude covariance matrix measured at $4 \mathrm{MHz}$ is diagonal with elements equal to one. It proves that the laser is only affected by the shot noise at this offset frequency. In this case, the laserfield can be approximated by a coherent state. On the contrary, the amplitude and phase matrices at $500 \mathrm{kHz}$ display correlations between spectral bands 
indicating that classical noise affects the laser dynamics. The next section presents how some information is extracted from those matrices.

It can be noted that the phase matrix at $4 \mathrm{MHz}$ is not purely diagonal as is the case for the amplitude one. This is a result of the limited sensitivity of the detection for the phase noise. Indeed, in a standard homodyne detection, the phase measured is the one of the weak beam. However, in our experiment, the weak beam is filtered and we aim at measuring the phase noise of the intense beam. Consequently, the normalization introduces losses in the detection, resulting in a decreased sensitivity as explained in Appendix C.

\section{EXTRACTING THE NOISE}

To retrieve the noise spectrum of the laser parameters, the covariance matrices are measured at offset frequencies from $200 \mathrm{kHz}$ to $4 \mathrm{MHz}$ by sweeping the frequency of the demodulating signal. We note $\Gamma^{x, p}(f)$ the amplitude and phase covariance matrices calculated at the offset frequency $f$. Measuring the covariance matrix is enough to recover all the information on the dynamics, assuming that the noises under study are Gaussian, which is a reasonable assumption in our experiment. Consequently, the noise relative to the shot noise for each of the four laser parameters, $\left(\delta \epsilon, \delta \omega_{c}, \delta \phi_{\mathrm{CEO}}=\right.$ $\left.\omega_{c} \delta \tau_{\mathrm{CEO}}, \delta \tau_{r}\right)$, defined in Sec. II A., can be extracted by mathematically projecting the covariance matrices on the corresponding modes defined in Eqs. (10) and (11). To do so, the noise modes need to be pixelized similarly to Eq. (13) for quadratures. We note those modes $\vec{u}$ mode. They are represented in Fig. 2(c). The resulting noise spectra are given by

$$
\begin{aligned}
\delta \epsilon(f) & =\left[\vec{u}_{\mathrm{amp}}^{T} \cdot \Gamma^{x}(f) \cdot \vec{u}_{\mathrm{amp}}\right]^{1 / 2}, \\
\delta \omega_{c}(f) & =\left[\vec{u}_{\text {cent-freq }}^{T} \cdot \Gamma^{x}(f) \cdot \vec{u}_{\text {cent-freq }}\right]^{1 / 2}, \\
\delta \phi_{\mathrm{CEO}}(f) & =\left[\vec{u}_{\mathrm{CEO}}^{T} \cdot \Gamma^{p}(f) \cdot \vec{u}_{\mathrm{CEO}}\right]^{1 / 2}, \\
\delta \tau_{r}(f) & =\left[\vec{u}_{\text {rep-rate }}^{T} \cdot \Gamma^{p}(f) \cdot \vec{u}_{\text {rep-rate }}\right]^{1 / 2},
\end{aligned}
$$

where $\vec{u}_{\text {mode }}^{T}$ is the transposed mode.

As the covariance matrices are normalized to the shot noise, all the fluctuations in the expressions above are expressed in units of shot noise. Figure 2(c) represents those fluctuations in $\mathrm{dB}$ relative to the shot noise as a function of the offset frequency, thus corresponding to the spectrum of the noise of each parameter. It can be seen that the dominant noises in this frequency range are the phase ones and mainly the noise on the CEO phase. The laser reaches the shot noise level around $3 \mathrm{MHz}$, meaning that technical noises are no longer affecting the laser. The peak around $1 \mathrm{MHz}$ corresponds to the relaxation oscillations of the laser. One can notice a discrepancy with respect to the shot noise at high frequency for the phase noises even though the phase noise is expected to reach the shot noise level at high frequencies. It is the consequence of the limited sensitivity of the measurement due to the renormalization, as explained before.

To further investigate the dynamics of the laser, the noise spectra represented in Fig. 2(c) can be converted into physical units. From Eqs. (10) and (11) and from the expressions of the NRSN Eq. (15), we thus obtain the following expressions for power spectral densities of the relative intensity noise $[\operatorname{RIN}(f)]$, the central frequency noise $\left[S_{\omega_{c}}(f)\right]$, the CEO frequency noise $\left[S_{f_{\mathrm{CEO}}}(f)\right]$, and the timing phase noise $\left[S_{\phi_{t}}(f)\right]$, with $\phi_{t}(f)=2 \pi f_{r} \delta \tau_{r}(f)$, together with their units:

$$
\begin{aligned}
\operatorname{RIN}(f) & =\left(\frac{\delta \epsilon(f)}{\sqrt{N_{0}}}\right)^{2} T_{m} \quad\left[\mathrm{~Hz}^{-1}\right], \\
S_{\omega_{c}}(f) & =\left(\frac{\Delta \omega}{\sqrt{N_{0}}} \delta \omega_{c}(f)\right)^{2} T_{m} \quad\left[\mathrm{rad}^{2} \mathrm{~s}^{-2} / \mathrm{Hz}\right], \\
S_{f_{\mathrm{CEO}}}(f) & =\left(\frac{f_{r}}{4 \pi \sqrt{N_{0}}} \delta \phi_{\mathrm{CEO}}(f)\right)^{2} T_{m} \quad\left[\mathrm{~Hz}^{2} / \mathrm{Hz}\right], \\
S_{\phi_{t}}(f) & =\left(\frac{\pi f_{r}}{\Delta \omega \sqrt{N_{0}}} \delta \tau_{r}(f)\right)^{2} T_{m} \quad\left[\mathrm{rad}^{2} / \mathrm{Hz}\right],
\end{aligned}
$$

where $N_{0}$ corresponds to the number of photons hitting the detector during the acquisition duration. It is given by $N_{0}=$ $P T_{m} / \hbar \omega_{c}$, where $P$ is the optical power before the BS of the homodyne detection $(P \simeq 5.5 \mathrm{~mW})$, and $T_{m}=1 / \mathrm{BW}$ the acquisition time with BW the bandwidth of the low-pass filter used in the detection chain after the demodulation $(\mathrm{BW}=$ $10 \mathrm{kHz}$ ). The spectra obtained are reproduced in Fig. 3. As a comparison, the timing phase noise and the RIN of a modelocked laser have been determined theoretically in Ref. [20], where similar noise levels have been found. Those spectra offer a quantitative measurement of the noise affecting the OFC, derived from a single measurement. These quantities are used in Sec. VI to track the origin of the noises, in particular, the intensity related dynamics of the CEO frequency.

\section{UNVEILING THE DYNAMICS FROM XP CORRELATIONS}

The experimental scheme allows the measurement of the amplitude and the phase quadratures simultaneously by taking, respectively, the sum and difference of the photocurrents of the 16 photodiodes after acquisition. Consequently, it is also possible to access the correlation matrix $\Gamma_{i j}^{x p}=$ $\left\langle\delta x\left(\Omega_{i}\right) \delta p\left(\Omega_{j}\right)\right\rangle$ between amplitude and phase. An example of such a matrix is represented in Fig. 4(a) for an offset frequency of $500 \mathrm{kHz}$ (4 MHz in inset).

These matrices show spectral correlations between the amplitude and the phase up to the $\mathrm{MHz}$ domain and no correlations above. To analyze this matrix, a SVD is performed. From this decomposition, the Schmidt number can be calculated. It is given by $K=\left(\sum_{n} \lambda_{n}^{2}\right)^{2} / \sum_{n} \lambda_{n}^{4}$, where the $\lambda_{n}$ 's are the singular values [35]. This parameter provides some information on the number of significant modes involved in the process. It is represented in Fig. 4(b) as a function of the offset frequency. The Schmidt number is equal to one up to $1 \mathrm{MHz}$, meaning that only one pair of modes, one in phase and one in amplitude, is necessary to reconstruct the correlations. Those two modes, represented in the same figure, are the singular modes. Because only one pair of modes is involved, we can assume that only one noise source is responsible for those correlations. As has been shown in various papers [24,26], it is expected to come from the fluctuations of the pump laser intensity. This is investigated in the next part. 

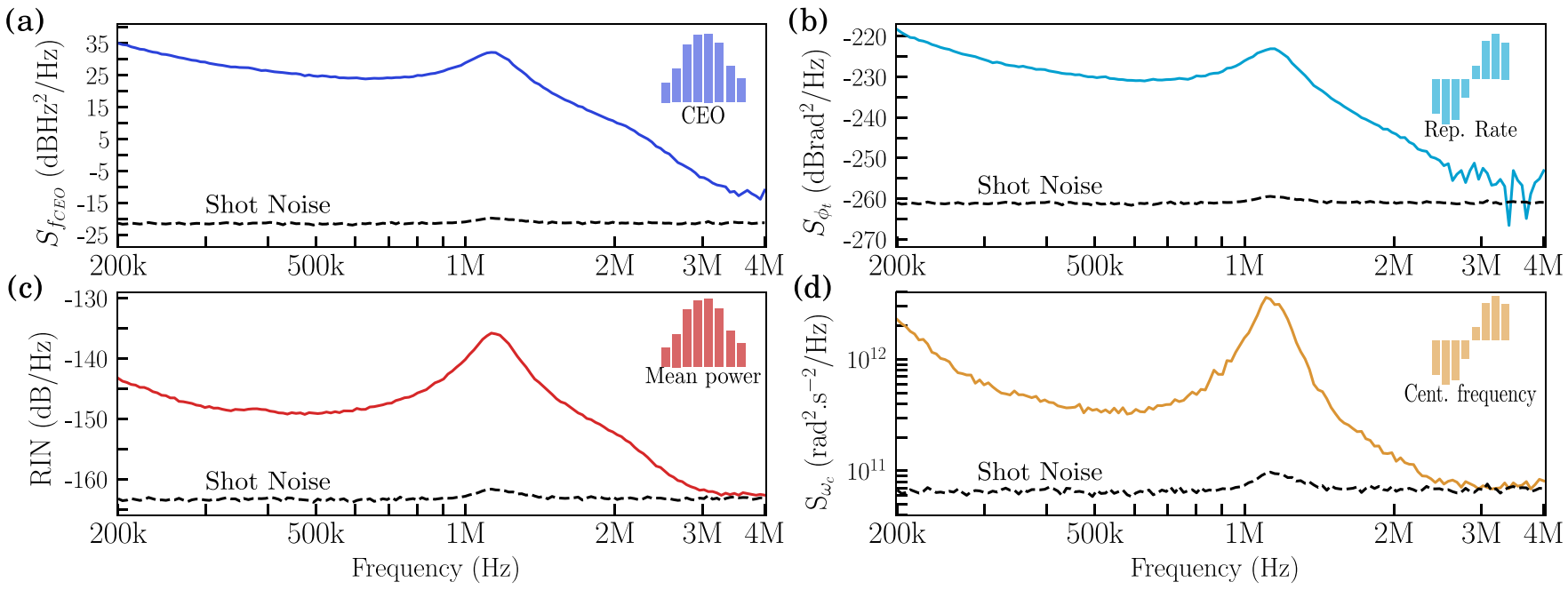

FIG. 3. Noise in physical units (a) CEO frequency noise, (b) timing jitter noise, (c) mean power noise, and (d) spectrum center frequency noise. The spectral mode corresponding to each quantity is reproduced in each plot. The shot noise is plotted as a dashed black line.

A qualitative understanding of the underlying processes can be obtained through the projection of the singular modes on the detection modes introduced earlier. The projection of the amplitude singular mode on the mean power and spectrum center frequency detection modes and the projection of the phase singular mode on the $\mathrm{CEO}$ and repetition rate detection modes are represented in Fig. 4(c). It can be seen that mainly three modes are coupled. In phase, only the CEO detection mode is coupled to the amplitude ones. Indeed, this was expected as it is well-known that intensity fluctuations, induced by pump power fluctuations, have a huge impact on the CEO of the laser and are even used to control it [36] as it is the case in this study. In amplitude, the singular mode is a linear combination of the mean power and spectrum center frequency modes. The dominant contribution comes from the fluctuations of spectrum center frequency. Despite the fact that there is a strong coupling between the CEO and the mean power fluctuations due to the Kerr effect, this contribution can be exceeded by the fluctuations of the spectrum center frequency. This is the case when there is a non- negligible residual GVD inside the laser cavity [24]. In the next section, we investigate those correlations with a simple model describing the intensity related dynamics of the CEO fluctuations.

\section{INTENSITY RELATED DYNAMICS: RECOVERING THE CEO FLUCTUATIONS}

As previously demonstrated, the dominant noise is the CEO frequency fluctuations. Moreover, only one noise source is expected to induce correlations between amplitude and phase as suggested by the SVD applied in the previous section. As a consequence, following the idea developed in Ref. [24], the intensity-related dynamics of the CEO frequency is investigated.

The CEO frequency is defined as follows:

$$
f_{\mathrm{CEO}}=\frac{f_{r}}{2 \pi} \Delta \phi_{\mathrm{CEO}}=\frac{\omega_{c}}{2 \pi}\left(1-\frac{v_{g}}{v_{\phi}}\right)
$$
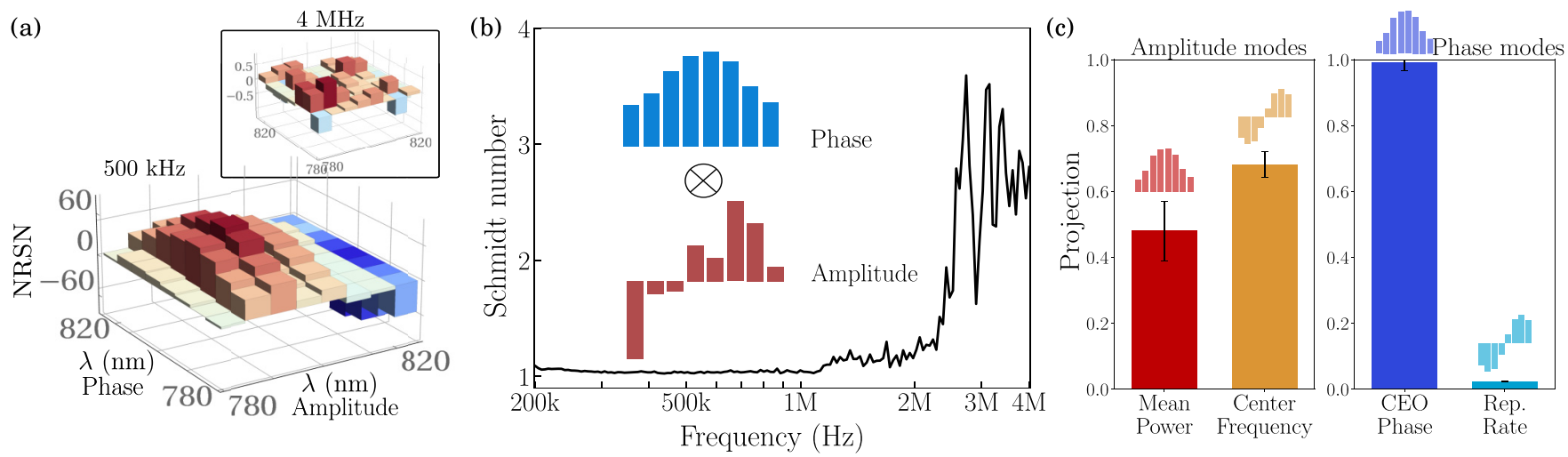

FIG. 4. Phase and amplitude correlations: (a) Correlation matrix between amplitude and phase at an offset frequency of $500 \mathrm{kHz}$. In inset the same but at $4 \mathrm{MHz}$ where the correlations vanish. (b) Schmidt decomposition of the correlation matrices. The Schmidt number as a function of the offset frequency is plotted together with the amplitude and phase singular modes for the frequencies where it is equal to one. (c) Projection of the amplitude and phase singular modes on the detection modes. 
where $\omega_{c}$ is the carrier frequency and $v_{g}$ and $v_{\phi}$ the average group and phase velocities defined by

$$
\begin{aligned}
\frac{c}{v_{g}} & =\bar{n}+\omega_{c} \frac{d \bar{n}}{d \omega}, \\
\frac{c}{v_{\phi}} & =\bar{n} .
\end{aligned}
$$

These velocities are averaged over the cavity length and contain the refractive index $\bar{n}=\bar{n}_{0}+\bar{n}_{2} I$, which is itself averaged over the cavity and includes the Kerr effect. An important point to note is that $\bar{n}$ depends on the intensity $I$ and on $\omega_{c}$, due to the dispersion, which itself depends on $I$. The central frequency can be affected by intensity fluctuations when an asymmetry between the gain and the loss profiles exists $[16,25]$. In that configuration, a change in the gain results in a frequency-pulling effect due to a shift of the equilibrium frequency.

Thus, the intensity dependence of the CEO frequency is given by

$$
\begin{aligned}
\frac{d f_{\mathrm{CEO}}}{d I}= & \frac{1}{2 \pi}\left[\frac{d \omega_{c}}{d I}\left(1-\frac{v_{g}}{v_{\phi}}\right)\right. \\
& \left.+\omega_{c} \frac{v_{g}}{v_{\phi}}\left(\frac{1}{v_{\phi}} \frac{d v_{\phi}}{d I}-\frac{1}{v_{g}} \frac{d v_{g}}{d I}\right)\right] .
\end{aligned}
$$

The model is derived with respect to the intracavity peak intensity $I$, which we calculate for a Fourier transformed limited pulse (the effect of dispersion is included later). However, in practice, we measure the fluctuations of the parameters with respect to the laser mean output power $P$. The conversion from $I$ to $P$ is given by

$$
\frac{d I}{d P}=\frac{2 \times 0.88}{f_{r} T_{\text {coupler }} \Delta t_{\text {pulse }} \pi w^{2}} .
$$

This quantity is determined from the parameters of the experiment: $f_{r}=155 \mathrm{MHz}, T_{\text {coupler }}=0.28$ the transmission of the output coupler, $\Delta t_{\text {pulse }}=22$ fs the pulse duration, and $w=10 \mu \mathrm{m}$ the waist in the crystal. The factor 0.88 comes from the hyperbolic secant shape of the pulse. Given those data, we find $d I / d P \simeq 5.8 \times 10^{-15} \mathrm{~m}^{-2}$.

From Eqs. (20) and (21), the CEO frequency fluctuations are given by

$$
\begin{aligned}
\delta f_{\mathrm{CEO}}= & \frac{1}{2 \pi}\left[\delta \omega_{c}\left(\left(1-\frac{v_{g}}{v_{\phi}}\right)+\omega_{c} \frac{v_{g}^{2}}{v_{\phi}} \mathrm{GVD}-\omega_{c} \frac{v_{g}}{c} \frac{\partial \bar{n}}{\partial \omega}\right)\right. \\
& \left.+\delta P \frac{d I}{d P} \frac{\omega_{c} v_{g}}{c}\left(\frac{v_{g}}{v_{\phi}} \omega_{c} \frac{\partial \bar{n}_{2}}{\partial \omega}-\bar{n}_{2}\left(1-\frac{v_{g}}{v_{\phi}}\right)\right)\right],
\end{aligned}
$$

where GVD is the average group velocity dispersion inside the cavity given by GVD $=\frac{d}{d \omega}\left(\frac{1}{v_{g}}\right)$.

From this expression, it is clear that the noise on the CEO frequency arises from the noise on the central frequency $\delta \omega_{c}$ and from the mean power fluctuations $\delta P$. The factor coupling the CEO frequency and the central frequency is composed of three terms. The first one comes from the dispersion in the laser, $\left(1-\frac{v_{g}}{v_{\phi}}\right)$, the second one from the GVD, and the last one is due to the dispersion of the Kerr effect $\frac{\partial \bar{n}}{\partial \omega}$. On the other hand, the term coupling the CEO frequency to the intensity is mainly due to the Kerr effect, as expected, via $\bar{n}_{2}$ and its dispersion.

To apply this formula, a few quantities need to be taken from the literature. We have $\bar{n}=1.00116, \bar{n}_{2}=$ $1.8 \times 10^{-23} \mathrm{~m}^{2} \mathrm{~W}^{-1}, \frac{\partial \bar{n}_{0}}{\partial \omega}=3.5 \times 10^{-21} \mathrm{~s}$, and $\frac{\partial \bar{n}_{2}}{\partial \omega}=3 \times$ $10^{-39} \mathrm{sm}^{2} \mathrm{~W}^{-1}$ [24]. Finally, the GVD of the laser needs to be estimated. Just after the output coupler, the duration of the pulse is $36 \mathrm{fs}$ for a $40-\mathrm{nm}$ spectrum, showing that the pulse is chirped. To have an estimation of the GVD inside the cavity, the passage through the output coupler needs to be taken into account. We estimate the dispersion introduced by $4 \mathrm{~mm}$ of silica of dispersion $\beta_{2}=36 \mathrm{fs}^{2} / \mathrm{mm}$. After the output coupler, the pulse duration is found to be $24 \mathrm{fs}$. The passage through the crystal also needs to be taken into account. However, as we derived the expression Eq. (22) of the fluctuations averaged over the cavity, this quantity varies, depending on where the noise arises in the laser cavity. Consequently, an uncertainty can be associated to the estimation of the GVD. Because the cavity is linear, each pulse goes through the crystal twice per round trip. Thus, to estimate the dispersion and its uncertainty, we calculate the GVD for one trip in the crystal and calculate the uncertainty associated to a pulse which has not yet traveled through the crystal or which has done a double pass. The crystal is made of $3 \mathrm{~mm}$ of sapphire of dispersion $\beta_{2}=58 \mathrm{fs}^{2} / \mathrm{mm}$. The resulting calculated GVD is $-280 \mathrm{fs}^{2}$ and the uncertainty is $\pm 50 \mathrm{fs}^{2}$. The calculation to estimate the GVD is detailed in the Appendices.

The model can now be applied to our measurements. We use the fluctuations of the mean power, $\delta P$, and of the central frequency, $\delta \omega_{c}$, measured experimentally thanks to our setup. For each offset frequency, the expected CEO frequency fluctuation is calculated using those values as well as the estimated GVD. This gives a spectrum for the CEO frequency fluctuations, which can be compared to the one measured with the setup. The resulting trace is reproduced in Fig. 5 alongside the measured CEO frequency fluctuations given by $\delta f_{\text {CEO }}=\sqrt{S_{f_{\text {CEO }}}}$. Good agreement is found between the model and the measured traces. The experimental data almost entirely fall in the uncertainty area up to $1 \mathrm{MHz}$. This agreement proves that the CEO dynamics is indeed related to the intensity fluctuations of the laser and that this feature is sufficient to explain it almost entirely. It confirms what was found with the SVD: the Schmidt number is equal to one as one noise source

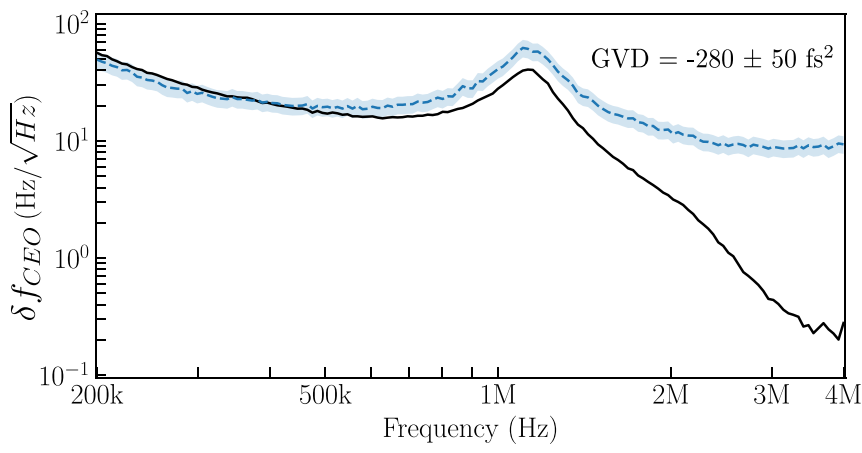

FIG. 5. Experimental versus model for the CEO noise: In dashed blue, the model from Eq. (22), the shaded zone corresponds to the uncertainty on the GVD determined in the laser. Plain line: Measured CEO frequency fluctuation given by $\delta f_{\text {CEO }}=\sqrt{S_{f_{\text {CEO }}}}$ from Eq. (16). 
TABLE I. Expressions and value of the ratio of contributions of the model to the measured CEO fluctuations. This ratio is averaged over the frequency range where the model is the most accurate, from $200 \mathrm{kHz}$ to $500 \mathrm{kHz}$. The first three lines are the contributions from the spectrum center frequency and the last one the contribution from the power fluctuations.

\begin{tabular}{llr}
\hline \hline Contribution & Expression & ratio $\left|c_{j} / \delta f_{\mathrm{CEO}, \exp }\right|$ \\
\hline Group velocity dispersion & $c_{1}=\frac{1}{2 \pi} \delta \omega_{c}\left(\omega_{c} \frac{v_{g}^{2}}{v_{\phi}} \mathrm{GVD}\right)$ & $9 \times 10^{-1}$ \\
Kerr effect dispersion & $c_{2}=\frac{1}{2 \pi} \delta \omega_{c}\left(\omega_{c} \frac{v_{g}}{c} \frac{\partial \bar{n}}{\partial \omega}\right)$ & $4 \times 10^{-2}$ \\
Dispersion & $c_{3}=\frac{1}{2 \pi} \delta \omega_{c}\left(1-\frac{v_{g}}{v_{\phi}}\right)$ & $5 \times 10^{-3}$ \\
Kerr effect & $c_{4}=\frac{1}{2 \pi} \delta P \frac{d I}{d P} \frac{\omega_{c} v_{g}}{c}\left(\frac{v_{g}}{v_{\phi}} \omega_{c} \frac{\partial \bar{n}_{2}}{\partial \omega}-\bar{n}_{2}\left(1-\frac{v_{g}}{v_{\phi}}\right)\right)$ & $2 \times 10^{-6}$ \\
\hline \hline
\end{tabular}

is responsible for the main dynamics. It also demonstrates the coupling between the CEO and the central frequency fluctuations which was found by the projection of the singular modes. Nonetheless, the model does not seem accurate above $1 \mathrm{MHz}$. One explanation is that the model does not take into account the shot noise and describes only the correlations between $\mathrm{CEO}$ and the amplitude modes. However, above $2 \mathrm{MHz}$ the amplitude noises get really close to the shot noise. This is particularly true for the noise of the center spectrum which turns out to be the main contribution to the CEO fluctuations as discussed later. Hence, the model is no longer relevant above $2 \mathrm{MHz}$. This could also be due to some additional filtering coming from the laser cavity or the detection scheme not taken into account in the model.

To further explore the model, the ratio of the contributions of the center frequency and mean power fluctuations, calculated from the model, to the measured CEO fluctuations are reported in Table I. It can be seen that the dominant contribution is the one coming from the spectrum center frequency. More precisely, it is the spectrum center frequency via the GVD which seems to be the dominant contribution. This is in agreement with the results found in Sec. V. The center spectrum detection mode has a higher contribution to the amplitude singular mode as it is directly coupled to the CEO frequency fluctuations via the GVD. This decomposition indicates that the mean power fluctuations have a really small direct effect on the CEO frequency. Note that in Fig. 4(c) there is still a significant contribution to the amplitude singular mode from the mean power because the fluctuations of center frequency are also coupled to the intensity fluctuations due to an asymmetry between the gain and loss profiles as explained before. Thus, the intensity has a significant impact on the CEO frequency only when there is a residual GVD inside the laser cavity. This has also been identified in Refs. [22,24]. The knowledge of this process can help improve the performance of frequency combs. To achieve a lower CEO noise, the noise of the pump laser can be reduced or the GVD of the laser cavity can be reduced so this intensity noise has a lower effect. In practice, the first option is probably the easiest to implement. Alternately, a better lock of the CEO frequency can be achieved by using a laser cavity with an appreciable amount of GVD.

\section{CONCLUSION}

We have presented a method enabling the characterization of the dynamics of an OFC with a single setup. From the amplitude and phase covariance matrices, the fluctuations of the laser parameters have been measured at the shot noise limit. The noises are also expressed in physical units to be compared to other studies. The noise levels measured are similar to what can be expected from the literature on OFC dynamics. In addition, we measured the amplitude-phase correlation matrix. Thanks to SVD, the underlying processes can be studied. We show that those correlations are mainly induced by the pump laser intensity fluctuations. Our analysis permits the identification of the coupling mechanisms. The fluctuations of the spectrum center frequency induced by the pump noise via frequency pulling is the main driving force of the CEO frequency noise. This is due to the GVD of the laser cavity which was estimated to be $-280 \mathrm{fs}^{2}$.

Using such a setup could help for the design of low noise frequency combs by characterizing their dynamics. This scheme could also be improved to implement feedback on the laser pump current as it is the main source of noise. Another improvement is to use a fiber-optic delay line to decorrelate the phase noise of the two arms of the homodyne detection. This scheme is easier to implement and can give access to noise at lower frequencies where the technical noise can be investigated.

\section{ACKNOWLEDGMENTS}

This work was supported by the Agence Nationale de la Recherche (LASAGNE ANR-16-ASTR-0010-03) and the Direction Générale de l'Armement.

\section{APPENDIX A: DETERMINATION OF THE LASER'S DISPERSION}

The aim is to determine the GVD parameter in the laser. The starting point is Eq. (2.118) in Ref. [37],

$$
\left\{\left(D_{g}+i D\right) \frac{d^{2}}{d t^{2}}+(g-l-i \psi)+(\gamma-i \delta)|a(t)|^{2}\right\} a(t)=0,
$$

with $g$ the gain per round trip and $\Omega_{g}^{2}$ the width of the gain in amplitude so $D_{g}=g / \Omega_{g}^{2}, l$ the losses per round trip, $\gamma$ the automodulation coefficient in amplitude, $\delta$ the self-phase modulation coefficient, $D$ the GVD, and $\psi$ the dephasing per round trip. We also use $U=2 a_{0}^{2} t_{p}$ the pulse energy as well as the normalized quantities $D_{n}=D / D_{g}, t_{p n}=U t_{p} / 2 D_{g}$. 
The solution of the equation for the mode-locked laser is a chirped hyperbolic secant:

$$
a(t)=a_{0}\left[\operatorname{sech}\left(\frac{t}{t_{p}}\right)\right]^{1+i \beta} .
$$

By putting Eq. (A2) in Eq. (A1), we find

$$
\begin{gathered}
g-l-i \psi+\frac{(1+i \beta)^{2}}{t_{p}^{2}}\left(D_{g}+i D\right)=0 \\
\left(D_{g}+i D\right) \frac{2+3 i \beta-\beta^{2}}{t_{p}^{2}}=(\gamma-i \delta) a_{0}^{2} .
\end{gathered}
$$

By multiplying the second equation by $D_{g} / t_{p}^{2}$ and identifying the real and imaginary parts, we have

$$
\begin{aligned}
& 2-\beta^{2}-3 \beta D_{n}=\gamma t_{p n} \\
& 3 \beta+2 D_{n}-\beta^{2} D_{n}=-\delta t_{p n} .
\end{aligned}
$$

From the second equation, we have the GVD depending on the duration of the pulse and its chirp:

$$
D=D_{n} \times D_{g}=D_{g} \frac{-\left(\delta t_{p n}+3 \beta\right)}{2-\beta^{2}} .
$$

Consequently, to determine the GVD, the duration of the pulse and its chirp in the cavity need to be known. At the output of the laser, the pulse duration has a duration of $36 \mathrm{fs}$ and a spectrum of $40 \mathrm{~nm}$ corresponding to a Fourier transform spectrum of $22 \mathrm{~nm}$. Consequently, the chirp parameter of the pulse is $\beta_{0}=\sqrt{(36 / 22)^{2}-1}=1.3$. The chirp parameter is defined so the electric field of the chirped pulse is given by $E(t)=E_{0} \exp \left(-\frac{1+i \beta}{2} \frac{t^{2}}{\Delta t_{0}}\right)$. The evolution of the duration and chirp of a chirped pulse going through $z \mathrm{~mm}$ of a dispersing medium of dispersion $\beta_{2}$ is given by

$$
\begin{aligned}
& \Delta t(z)=\Delta t_{0} \sqrt{\left(1+\frac{\beta_{2} z \beta_{0}}{\Delta t_{0}}\right)^{2}+\left(\frac{\beta_{2} z}{\Delta t_{0}^{2}}\right)^{2}}, \\
& \beta(z)=\beta_{0}+\frac{\beta_{2} z \beta_{0}^{2}}{\Delta t_{0}^{2}}+\frac{\beta_{2} z}{\Delta t_{0}^{2}} .
\end{aligned}
$$

\section{APPENDIX B: HOMODYNE DETECTION}

The fields $E_{ \pm}^{(+)}$at each output of the BS can be expressed as

$$
E_{ \pm}^{(+)}(t)=\frac{E_{s}^{(+)}(t) \pm E_{\mathrm{ref}}^{(+)}(t)}{\sqrt{2}}
$$

where $E_{s}^{(+)}(t)=\mathcal{E}_{0} a_{s}(t) e^{-i \omega_{0} t}$ is the field coming from the intense beam and $E_{\text {ref }}^{(+)}(t)=\mathcal{E}_{0} a_{\text {ref }}(t) e^{-i \omega_{0} t}$ from the weak one. The instantaneous intensity detected at each BS output, $S_{ \pm}$, given according to $I(t)=2 \epsilon_{0} n c\left|E^{(+)}(t)\right|^{2}$ in $\left(\mathrm{J} / \mathrm{s} / \mathrm{m}^{2}\right)$, is

$$
\begin{aligned}
S_{ \pm}(t) & =\frac{I_{s}(t)}{2}+\frac{I_{\mathrm{ref}}(t)}{2} \\
& \pm n c \epsilon_{0}\left\{E_{s}^{(+)}(t) E_{\mathrm{ref}}^{(-)}(t)+E_{s}^{(-)}(t) E_{\mathrm{ref}}^{(+)}(t)\right\} .
\end{aligned}
$$

At this point, it is more convenient to work in the frequency domain. The intensity at each output can thus be written

$$
S_{ \pm}=\frac{I_{s}}{2}+\frac{I_{\mathrm{ref}}}{2} \pm \mathcal{E}_{0}^{2} n c \epsilon_{0}\left\{a_{s}(\Omega) a_{\mathrm{ref}}^{\star}(\Omega)+a_{s}^{\star}(\Omega) a_{\mathrm{ref}}(\Omega)\right\} .
$$

The envelope of the field can be decomposed in a modulus and phase term,

$$
\begin{aligned}
a_{\mathrm{ref}}(\Omega) & =\left|a_{\mathrm{ref}}(\Omega)\right| e^{i \phi_{\mathrm{ref}}(\Omega)}=\alpha_{\mathrm{ref}}(\Omega) e^{i \phi_{\mathrm{ref}}(\Omega)}, \\
a_{s}(\Omega) & =\left|a_{s}(\Omega)\right| e^{i \phi_{s}(\Omega)}=\alpha_{s}(\Omega) e^{i \phi_{s}(\Omega)},
\end{aligned}
$$

where $\phi_{j}(\Omega)$ is the phase of the reference or signal pulse.

Summing the signal from both detectors gives access to an amplitude measurement of the signal. Indeed, because the signal field is stronger than the reference one, the intensity fluctuations measured are mainly the signal beam ones. The signal $\mathcal{I}_{+}=S_{+}+S_{-}$can be written as

$$
\begin{aligned}
\mathcal{I}_{+}(\Omega) & =\mathrm{I}_{s}+\mathrm{I}_{\mathrm{ref}} \\
& =2 \epsilon_{0} n c \mathcal{E}_{0}^{2}\left(\left|a_{s}(\Omega)\right|^{2}+\left|a_{\mathrm{ref}}(\Omega)\right|^{2}\right) \\
& =2 \epsilon_{0} n c \mathcal{E}_{0}^{2}\left(\alpha_{s}^{2}(\Omega)+\alpha_{\mathrm{ref}}^{2}(\Omega)\right) .
\end{aligned}
$$

Consequently, the fluctuations of the sum of the signals are given by

$$
\delta \mathcal{I}_{+}(\Omega) \propto \alpha_{s}(\Omega) \delta \alpha_{s}(\Omega) \Rightarrow \delta \mathcal{I}_{+}(\Omega) \propto \alpha_{s}(\Omega) \delta x_{s}(\Omega) .
$$

Taking the difference of the signals leads to a measurement of the relative phase between the two fields. With the decompositions Eqs. (B4) and (B5), the difference of the output intensities is

$$
\mathcal{I}_{-}(\Omega)=\alpha_{s}(\Omega) \mathcal{E}_{0}^{2} n c \epsilon_{0}\left\{\alpha_{\mathrm{ref}}(\Omega) e^{i \phi(\Omega)}+\alpha_{\mathrm{ref}}(\Omega) e^{-i \phi(\Omega)}\right\},
$$

where $\phi(\Omega)=\phi_{s}(\Omega)-\phi_{\text {ref }}(\Omega)$ is the relative phase between the two pulses.

In what follows, we are interested in studying the phase fluctuations of the signal. To do so, the field's $\left(E^{(+)}(\Omega)=\right.$ $\left.\mathcal{E}_{0} \alpha(\Omega) e^{i \phi(\Omega)}\right)$ fluctuations, $\delta E$, can be expanded to the first order:

$$
\delta E^{(+)}(\Omega)=\mathcal{E}_{0} e^{i \phi(\Omega)}(\delta \alpha(\Omega)+i \delta \phi(\Omega) \alpha(\Omega)) .
$$

As the signal is intense, the amplitude fluctuation can be neglected and the field expressed by its mean value $\alpha_{s}$. With expressions Eq. (B8), the fluctuations of the difference of output intensities can be written:

$$
\begin{gathered}
\delta \mathcal{I}_{-}(\Omega)=2 \alpha_{s} \mathcal{E}_{0}^{2} n c \epsilon_{0}\left\{\delta \alpha_{\mathrm{ref}}(\Omega) \cos \phi(\Omega)\right. \\
\left.-\alpha_{\mathrm{ref}}(\Omega) \delta \phi(\Omega) \sin \phi(\Omega)\right\}
\end{gathered}
$$

The phase difference between the reference and the signal needs to be the same for all frequencies and set to $\pm \frac{\pi}{2}$ by locking the relative path of the two arms. This locking point ensures retrieving the phase fluctuations when the difference of the signals is taken. Finally, as we are interested in the signal phase fluctuations only, the phase fluctuations of the reference have to be low. This can be accomplished by filtering the reference field so $\delta \phi(\Omega)=\delta \phi_{s}$.

Taking into account all those effects, the final signal is given by

$$
\delta \mathcal{I}_{-}(\Omega) \propto \alpha_{s}(\Omega) \alpha_{\text {ref }}(\Omega) \delta \phi_{s}(\Omega) \Rightarrow \delta \mathcal{I}_{-}(\Omega) \propto \alpha_{s}(\Omega) \delta p_{\text {ref }}
$$

With this detection scheme, the phase and amplitude fluctuations can be retrieved. To measure both amplitude and phase fluctuations at the same time to study their correlations, they are measured by collecting the signal of each detector and numerically calculating the sum and difference. 


\section{APPENDIX C: NORMALIZATION COVARIANCE MATRICES:}

Once the data are acquired, they need to be normalized before interpretation. The first step is to normalize the data with respect to the shot noise.

According to Eq. (B6) and taking into account the dark noise as well as a pixel-dependent gain, the measured intensity can be expressed as

$$
\delta \mathcal{I}_{+, i}=g_{i} \delta x_{s, i}+d_{i},
$$

where $g_{i}$ is a variable gain, $d_{i}$ the dark noise, and $i$ is the pixel's index. Taking the covariance of the measured intensity, assuming no correlations between the signal and the dark noise, leads to

$$
\operatorname{cov}\left[\delta \mathcal{I}_{+}\right]_{i, j}=g_{i} g_{j} \operatorname{cov}\left[\delta x_{s}\right]_{i, j}+\operatorname{cov}[d]_{i, j} .
$$

To determine the gain factor, the variance of the signal is calculated at a high frequency so the only noise measured is the shot noise, i.e., $\operatorname{var}\left[\delta x_{s}\right]_{i \text {,shot }}=1$. Thus, $g_{i}$ is given by

$$
g_{i}=\sqrt{\operatorname{var}\left[\delta \mathcal{I}_{+}\right]_{i, \text { shot }}-\operatorname{var}[d]_{i}} .
$$

Finally, the amplitude quadrature is given by

$$
\operatorname{cov}\left[\delta x_{s}\right]_{i, j}=\frac{\operatorname{cov}\left[\delta \mathcal{I}_{+}\right]_{i, j}-\operatorname{cov}[d]_{i, j}}{g_{i} g_{j}} .
$$

The normalization is slightly more complicated for the phase quadrature. As seen in Eq. (B10), the phase fluctuations measured, $\delta \mathcal{I}_{-, i} \propto \alpha_{s, i} \alpha_{\text {ref }, i} \delta \phi_{s, i}$, are proportional to the number of photons in the reference field, which is weaker than the signal $\left(\frac{\alpha_{s}^{2}}{\alpha_{\text {ref }}^{2}} \simeq 50\right)$. Consequently, the phase fluctuations measured are not directly the fluctuations from the signal but the ones from the reference. It can be seen as the signal fluctuations measured after a loss channel. Those losses can easily be modeled by a BS where one input is the signal and the other one the vacuum. This BS would have a reflectivity $r_{i}=\frac{\alpha_{\text {ref }, i}}{\alpha_{s, i}}$ and a transmission $t_{i}=\sqrt{1-\frac{\alpha_{\mathrm{ref}, i}^{2}}{\alpha_{s, i}^{2}}}$. Thus, the measured phase fluctuations and signal ones are related by

$$
\delta p_{\mathrm{ref}, i}=\frac{\alpha_{\mathrm{ref}, i}}{\alpha_{s, i}} \delta p_{s, i}+\sqrt{1-\frac{\alpha_{\mathrm{ref}, i}^{2}}{\alpha_{s, i}^{2}}} \delta p_{v, i}=r_{i} \delta p_{s, i}+t_{i} \delta p_{v, i}
$$

where $\delta p_{v}$ are the phase fluctuations of the vacuum.

As previously, the intensity fluctuations are related to the phase ones by

$$
\delta \mathcal{I}_{-, i}=g_{i} \delta p_{\mathrm{ref}, i}+d_{i},
$$

where, here again, $g_{i}$ is a variable gain and $d_{i}$ the dark noise. They are not necessarily the same as for the amplitude fluctuations but the same notation is used for simplicity.

Using the previous expression for $\delta p_{\text {ref }}$, the intensity fluctuations can be written as

$$
\delta \mathcal{I}_{-, i}=g_{i} r_{i} \delta p_{s, i}+g_{i} t_{i} \delta p_{v, i}+d_{i} .
$$

As none of those contributions are correlated, the covariance is given by

$$
\begin{aligned}
\operatorname{cov}\left[\delta \mathcal{I}_{-}\right]_{i, j}= & g_{i} g_{j} r_{i} r_{j} \operatorname{cov}\left[\delta p_{s}\right]_{i, j} \\
& +e s g_{i} g_{j} t_{i} t_{j} \operatorname{cov}\left[\delta p_{v}\right]_{i, j}+\operatorname{cov}[d]_{i, j} .
\end{aligned}
$$

Once again, to determine the gain factor, the variance of the signal is measured at a high frequency so $\operatorname{var}\left[\delta p_{s}\right]_{i, \text { shot }}=$ 1. Furthermore, $\operatorname{var}\left[\delta p_{v}\right]_{i}=1$, as the vacuum is uncorrelated. Finally, knowing that $r_{i}^{2}+t_{i}^{2}=1$, the same equation as Eq. (C3) is found for gain:

$$
g_{i}=\sqrt{\operatorname{var}\left[\delta \mathcal{I}_{-}\right]_{i, \text { shot }}-\operatorname{var}[d]_{i}} .
$$

Eventually, the covariance matrix for the signal phase fluctuations is given according to

$$
\operatorname{cov}\left[\delta p_{s}\right]_{i, j}=\frac{\operatorname{cov}\left[\delta \mathcal{I}_{-}\right]_{i, j}-\operatorname{cov}[d]_{i, j}}{g_{i} g_{j} r_{i} r_{j}}-\frac{t_{i} t_{j}}{r_{i} r_{j}} I d,
$$

where $I d$ is the identity matrix. As a matter of fact, the covariance of the vacuum fluctuations is the identity matrix because no correlations exist between the different frequency ranges.
[1] S. A. Diddams, D. J. Jones, J. Ye, S. T. Cundiff, J. L. Hall, J. K. Ranka, R. S. Windeler, R. Holzwarth, T. Udem, and T. W. Hänsch, Direct Link Between Microwave and Optical Frequencies with a 300 Thz Femtosecond Laser Comb, Phys. Rev. Lett. 84, 5102 (2000).

[2] T. Udem, R. Holzwarth, and T. W. Hänsch, Optical frequency metrology, Nature 416, 233 (2002).

[3] T. Udem, S. A. Diddams, K. R. Vogel, C. W. Oates, E. A. Curtis, W. D. Lee, W. M. Itano, R. E. Drullinger, J. C. Bergquist, and L. Hollberg, Absolute Frequency Measurements of the $\mathrm{Hg}^{+}$and Ca Optical Clock Transitions with a Femtosecond Laser, Phys. Rev. Lett. 86, 4996 (2001).

[4] A. Shelkovnikov, R. J. Butcher, C. Chardonnet, and A. AmyKlein, Stability of the Proton-To-Electron Mass Ratio, Phys. Rev. Lett. 100, 150801 (2008).

[5] H. Fleurbaey, S. Galtier, S. Thomas, M. Bonnaud, L. Julien, F. Biraben, F. Nez, M. Abgrall, and J. Guéna, New Measurement of the $1 s-3 s$ Transition Frequency of Hydrogen: Contribution to the Proton Charge Radius Puzzle, Phys. Rev. Lett. 120, 183001 (2018).

[6] A. Grinin, A. Matveev, D. C. Yost, L. Maisenbacher, V. Wirthl, R. Pohl, T. W. Hänsch, and T. Udem, Two-photon frequency comb spectroscopy of atomic hydrogen, Science 370, 1061 (2020).

[7] S. A. Diddams, L. Hollberg, and V. Mbele, Molecular fingerprinting with the resolved modes of a femtosecond laser frequency comb, Nature 445, 627 (2007).

[8] N. Picqué and T. W. Hänsch, Frequency comb spectroscopy, Nat. Photonics 13, 146 (2019).

[9] F. R. Giorgetta, W. C. Swann, L. C. Sinclair, E. Baumann, I. Coddington, and N. R. Newbury, Optical two-way time and frequency transfer over free space, Nat. Photonics 7, 434 (2013).

[10] J. Guéna, S. Weyers, M. Abgrall, C. Grebing, V. Gerginov, P. Rosenbusch, S. Bize, B. Lipphardt, H. Denker, N. Quintin, 
S. M. F. Raupach, D. Nicolodi, F. Stefani, N. Chiodo, S. Koke, A. Kuhl, F. Wiotte, F. Meynadier, E. Camisard, C. Chardonnet, Y. L. Coq, M. Lours, G. Santarelli, A. Amy-Klein, R. L. Targat, O. Lopez, P. E. Pottie, and G. Grosche, First international comparison of fountain primary frequency standards via a long distance optical fiber link, Metrologia 54, 348 (2017).

[11] K. Predehl, G. Grosche, S. M. F. Raupach, S. Droste, O. Terra, J. Alnis, T. Legero, T. W. Hänsch, T. Udem, R. Holzwarth, and H. Schnatz, A 920-kilometer optical fiber link for frequency metrology at the 19th decimal place, Science 336, 441 (2012).

[12] I. Coddington, W. C. Swann, L. Nenadovic, and N. R. Newbury, Rapid and precise absolute distance measurements at long range, Nat. Photonics 3, 351 (2009).

[13] P. Jian, O. Pinel, C. Fabre, B. Lamine, and N. Treps, Real-time displacement measurement immune from atmospheric parameters using optical frequency combs, Opt. Express 20, 27133 (2012).

[14] R. A. McCracken, J. M. Charsley, and D. T. Reid, A decade of astrocombs: Recent advances in frequency combs for astronomy, Opt. Express 25, 15058 (2017).

[15] H. A. Haus and Y. Lai, Quantum theory of soliton squeezing: A linearized approach, J. Opt. Soc. Am. B 7, 386 (1990).

[16] H. A. Haus and A. Mecozzi, Noise of mode-locked lasers, IEEE J. Quantum Electron. 29, 983 (1993).

[17] F. Glerean, G. Jarc, A. Marciniak, F. Giusti, G. Sparapassi, A. Montanaro, E. M. Rigoni, J. O. Tollerud, and D. Fausti, Time-resolved multimode heterodyne detection for dissecting coherent states of matter, Opt. Lett. 45, 3498 (2020).

[18] G. Brajato, L. Lundberg, V. Torres-Company, M. Karlsson, and D. Zibar, Bayesian filtering framework for noise characterization of frequency combs, Opt. Express 28, 13949 (2020).

[19] R. Paschotta, Noise of mode-locked lasers (Part I): Numerical model, Appl. Phys. B 79, 153 (2004).

[20] R. Paschotta, Noise of mode-locked lasers (Part II): Timing jitter and other fluctuations, Appl. Phys. B 79, 163 (2004).

[21] R. Paschotta, A. Schlatter, S. Zeller, H. Telle, and U. Keller, Optical phase noise and carrier-envelope offset noise of modelocked lasers, Appl. Phys. B 82, 265 (2006).

[22] N. R. Newbury and B. R. Washburn, Theory of the frequency comb output from a femtosecond fiber laser, IEEE J. Quantum Electron. 41, 1388 (2005).

[23] N. R. Newbury and W. C. Swann, Low-noise fiber-laser frequency combs (Invited), J. Opt. Soc. Am. B 24, 1756 (2007).

[24] K. W. Holman, R. J. Jones, A. Marian, S. T. Cundiff, and J. Ye, Detailed studies and control of intensity-related dynamics of femtosecond frequency combs from mode-locked Ti: Sapphire lasers, IEEE J. Sel. Top. Quantum Electron. 9, 1018 (2003).

[25] C. R. Menyuk, J. K. Wahlstrand, J. Willits, R. P. Smith, T. R. Schibli, and S. T. Cundiff, Pulse dynamics in modelocked lasers: Relaxation oscillations and frequency pulling, Opt. Express 15, 6677 (2007).

[26] J. Wahlstrand, J. Willits, T. Schibli, C. Menyuk, and S. Cundiff, Quantitative measurement of timing and phase dynamics in a mode-locked laser, Opt. Lett. 32, 3426 (2007).

[27] D. J. Jones, S. A. Diddams, J. K. Ranka, A. Stentz, R. S. Windeler, J. L. Hall, and S. T. Cundiff, Carrier-envelope phase control of femtosecond mode-locked lasers and direct optical frequency synthesis, Science 288, 635 (2000).

[28] D. Hou, C.-C. Lee, Z. Yang, and T. Schibli, Timing jitter characterization of mode-locked lasers with $1 \mathrm{zs} / \sqrt{\mathrm{Hz}}$ resolution using a simple optical heterodyne technique, Opt. Lett. 40, 2985 (2015).

[29] M. Wada, F.-L. Hong and H. Inaba, Frequency noise measurement and its uncertainty estimation of an optical frequency comb using a delay line interferometer, Meas. Sci. Technol. 31, 125012 (2020).

[30] H. Tian, W. Yang, D. Kwon, R. Li, Y. Zhao, J. Kim, Y. Song, and $\mathrm{M}$. Hu, Optical frequency comb noise spectra analysis using an asymmetric fiber delay line interferometer, Opt. Express 28, 9232 (2020).

[31] R. Schmeissner, V. Thiel, C. Jacquard, C. Fabre, and N. Treps, Analysis and filtering of phase noise in an optical frequency comb at the quantum limit to improve timing measurements, Opt. Lett. 39, 3603 (2014).

[32] G. Grynberg, A. Aspect, and C. Fabre, Introduction to Quantum Optics: From the Semi-Classical Approach to Quantized Light (Cambridge University Press, Cambridge, UK, 2010).

[33] S. De, V. Thiel, J. Roslund, C. Fabre, and N. Treps, Modal analysis for noise characterization and propagation in a femtosecond oscillator, Opt. Lett. 44, 3992 (2019).

[34] A. Monmayrant, S. Weber, and B. Chatel, A newcomer s guide to ultrashort pulse shaping and characterization, J. Phys. B: At., Mol. Opt. Phys. 43, 103001 (2010).

[35] A. Christ, K. Laiho, A. Eckstein, K. N. Cassemiro, and C. Silberhorn, Probing multimode squeezing with correlation functions, New J. Phys. 13, 033027 (2011).

[36] L. Xu, T. W. Hänsch, C. Spielmann, A. Poppe, T. Brabec, and F. Krausz, Route to phase control of ultrashort light pulses, Opt. Lett. 21, 2008 (1996).

[37] A. Weiner, Ultrafast Optics (John Wiley \& Sons, Hoboken, NJ, 2011), Vol. 72. 